\title{
Functional and radiological outcome of modified spinous process osteotomy (MSPO) technique in decompressive surgery for lumbar canal stenosis
}

\author{
Kumar Chandan ${ }^{1, *}$, Jim F. Vellara ${ }^{2}$, Harshal B. Bamb ${ }^{3}$, Jai Krishnan K.S ${ }^{4}$, Anubhav Sharma ${ }^{4}$ \\ ${ }^{1}$ Spine Fellow, ${ }^{2}$ Senior Consultant, ${ }^{4}$ Associate Professor, ${ }^{5}$ Junior Resident, Dept. of Orthopaedics, Amrita Institute of Medical

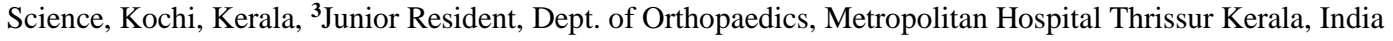 \\ *Corresponding Author: Kumar Chandan \\ Email: drkumarchandan@gmail.com
}

\begin{abstract}
Introduction: The Modified Spinous Process Osteotomy (MSPO) surgery is done for degenerative spinal canal stenosis which gives adequate exposure and avoid iatrogenic instability and long segment fusion following conventional laminectomy. The objective of this article is to evaluate clinical and radiological results of the MSPO.

Materials and Methods: Total 20 patients (13 male and 7 female) were included and studied prospectively with multilevel degenerative lumbar canal stenosis that were operated for posterior decompression by MSPO and laminectomy after detailed clinical and radiological work up. Regular follow up was done at 6 weeks 3 months and 1 year, clinical assessment for leg pain and back pain done by VAS, functional measurement of disability by Oswestry Disability Index (ODI) done. Dynamic view Xray of lumbar spine taken and compared to the preoperative X-ray. CT scan was taken at 1 year post op to assess the union of spinous process.

Results: All patients were followed up for 3 years. All patients recovered completely from thigh and leg pain and no patients had neurological deterioration. VAS for LBA and leg pain improved significantly along with significant improvement in ODI. The overall results were good to excellent in $93 \%$ of the patients, fair in $7 \%$ and no poor outcome. All patients were satisfied with the outcome at the last follow-up. The osteotomised spinous process eventually united with the retained laminar bridge in all patients. Conclusion: A less invasive and less techniquelly demanding MSPO technique provides better exposure and visualization, retaining median structures with least disturbance of kinematics of the lumbar spine. This procedure gives advantages for early ambulation and normalisation of patient's lifestyle and early return to work.
\end{abstract}

Keywords: MSPO, Decompression, Laminectomies, Lumbar canal stenosis.

\section{Introduction}

Lumbar canal stenosis is defined as an anatomical or functional narrowing of the osteoligamentus vertebral canal with or without the intervertebral foramina causing direct compression or indirect compromise of dural sac, the caudal nerve roots and their blood supply, enough to cause signs and symptoms. ${ }^{1}$ Decompressive laminectomy has been widely used as an operative treatment for lumbar canal stenosis. $^{2}$ A commonly used surgical decompression procedure for this type of spinal stenosis is a single level facet-sparing laminectomy. Although the impinged nerves are decompressed and neurological symptoms, such as sciatica, claudication, and motor, sensory and reflex activities, improve following lumbar laminectomy $^{3}$, the anatomically destructive character of this technique obviously affects spinal biomechanics. In fact, laminectomy can lead to symptomatic postoperative lumbar clinical segment instability., Long segment fusions have been done by some surgeon to reduce post operative instability, however, such operations leads to loss of motion of lumbar spine and predispose the spine for adjacent level degeneration ${ }^{6-9}$ It is important in the treatment of spinal stenosis to achieve adequate spinal decompression while maintaining the spinal stability. ${ }^{10}$

The preservation of posterior spinal elements associated with minimally invasive non fusion surgery technique could minimize the risk of developing instability and adjascent segment changes in long segment fusion. The integrity of posterior ligament complex is preserved by sparing the median structures integrity, i.e. spinous process, intraspinous ligaments and supraspinous ligaments, by osteotomizing the base of spinous process and retracting the whole midline structure to opposite side for the decompressive procedure. After completion of decompression, midline structure repositioned, osteotomized end of spinous process will get in apposition after tight closure of paraspinal muscle. We described this technique as modified spinous process osteotomy (MSPO).

\section{Materials and Methods}

The material for this study comprised of single or multilevel degenerative lumbar canal stenosis age group of 45-70 years operated at Amrita Institute of medical Science, Kochi, Kerala from 2013-2016. In our study total 20 patients (13 male and 7 female) were included and studied prospectively with multilevel degenerative lumbar canal stenosis that were operated for posterior decompression by modified spinous process osteotomy and laminectomy. They presented to our OPD with pain in lower limbs while walking (neurogenic claudication), attaining a stooping posture to get some pain relief while walking, with radiating pains in lower limb. Each patient had symptoms for 4-6 months and were initially treated conservatively with NSAIDs and short course of oral steroids for 2-3 months. As the radicular pain was 
getting worse, they were planned for MSPO. All patients were admitted one day prior to surgery and detailed clinical and radiological work up was done. Pre-op dynamic view X-ray were taken to confirm instability. Magnetic resonance imaging (MRI) was done for all patients. Pre operative subjective assessment of leg pain and back pain done by Visual Analog Scale (VAS) and Oswestry Disability Index (ODI).

Common comorbidities in the elderly group were hypertension in 7 patients $(\mathrm{M}=5, \mathrm{~F}=2)$, Diabetes in 8 patients $(M=3, F=5)$. All patients were managed for comorbidities by the physician before the surgery. We performed surgery on patients with severe leg pain with or without motor weakness with reduce walking (Claudication) distance. We have excluded the patients with traumatic, metabolic and congenital causes of LCS, patients with pre-op instability and previous history of any lumbar surgery (Table 1).

\section{Table 1}

\begin{tabular}{|c|c|}
\hline Inclusion Criteria & Exclusion Criteria \\
\hline 1. Degenerative lumbar canal stenosis (LCS) & Cogenital and traumatic causes of LCS \\
\hline 2. Failed conservative syndrome & 2. Metabolic causes of LCS \\
\hline \multirow[t]{2}{*}{ 3. Age $45-70$ years } & 3. $\quad$ Preoperative instability \\
\hline & 4. Previous lumbar spine surgery \\
\hline
\end{tabular}

Operative Technique: This surgery was performed in prone position on Wilson's frame. A midline incision was made through the skin and subcutaneous tissues (Fig. 1). Length of skin incision was depends on the number of involved level. For single level stenosis $3 \mathrm{~cm}$ incision was put and the maximum length of skin incision was $10-12 \mathrm{~cm}$ for multilevel stenosis. Unilateral paraspinal muscles reflected from Median structures sparing supraspinous and interspinous ligaments, and spinous processes. Self retaining retractor placed (Fig. 2). Spinous processes osteotomy were done near the base of spinous process (Fig. 4). The number of spinous processes cut depends on the number of levels to be decompressed. For a 4 level decompression, 3 spinous processes were cut and for single level 2 spinous process osteotomy done for better exposure and mobilization of midline structure. The required spinous processes attached with their supraspinous and interspinous ligament were retracted to one side (Fig. 5).

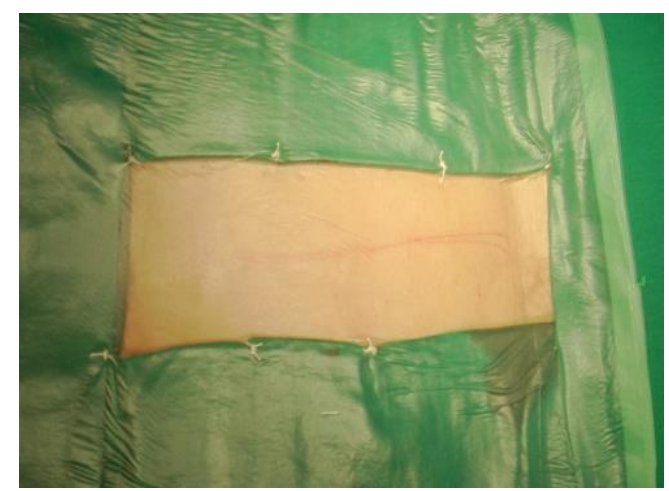

Fig. 1

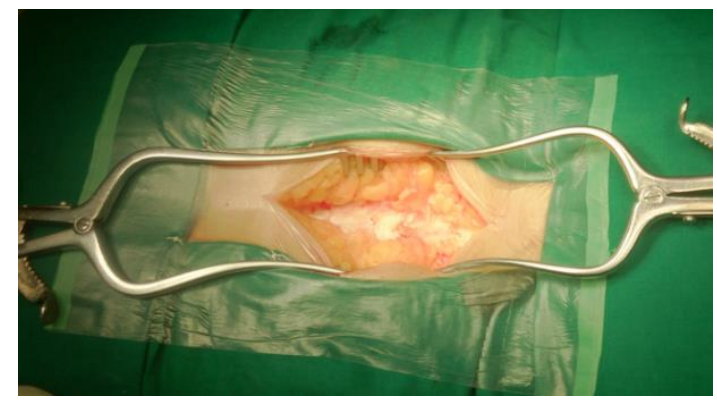

Fig. 2

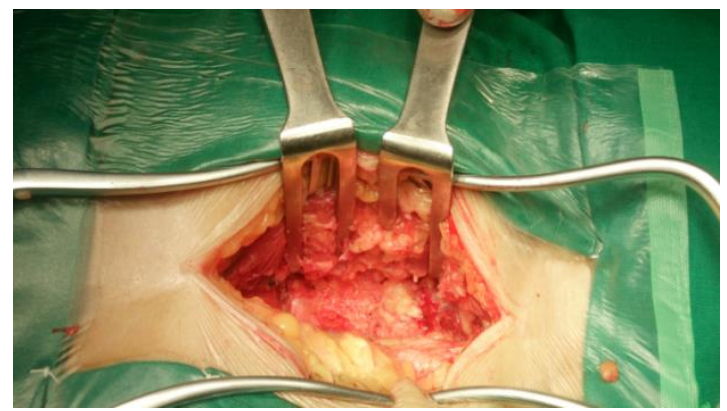

Fig. 3

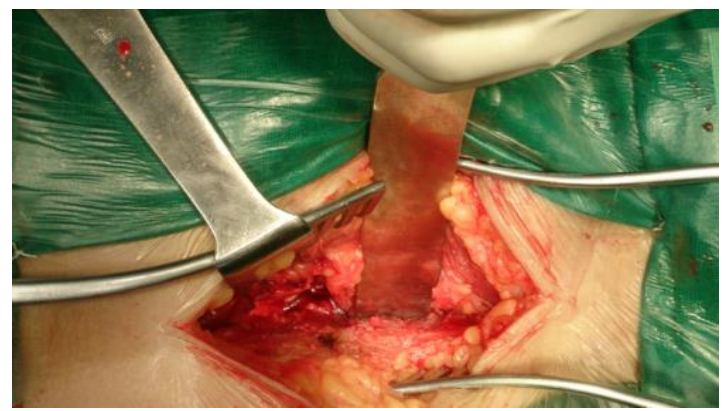

Fig. 4 


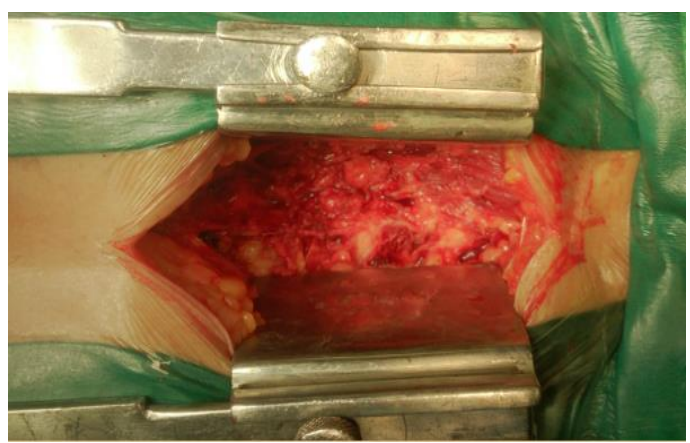

Fig. 5

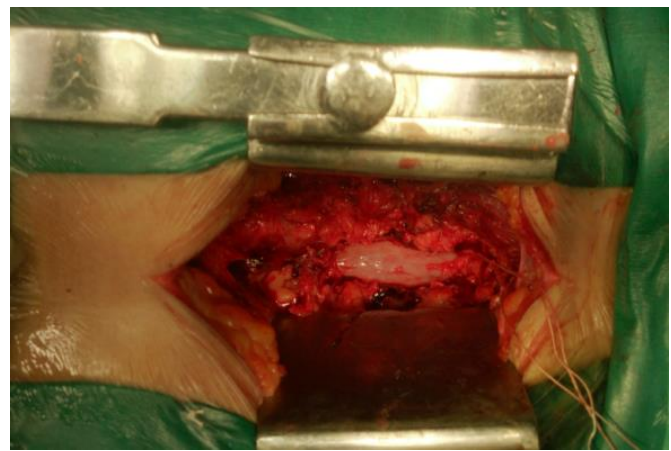

Fig. 6
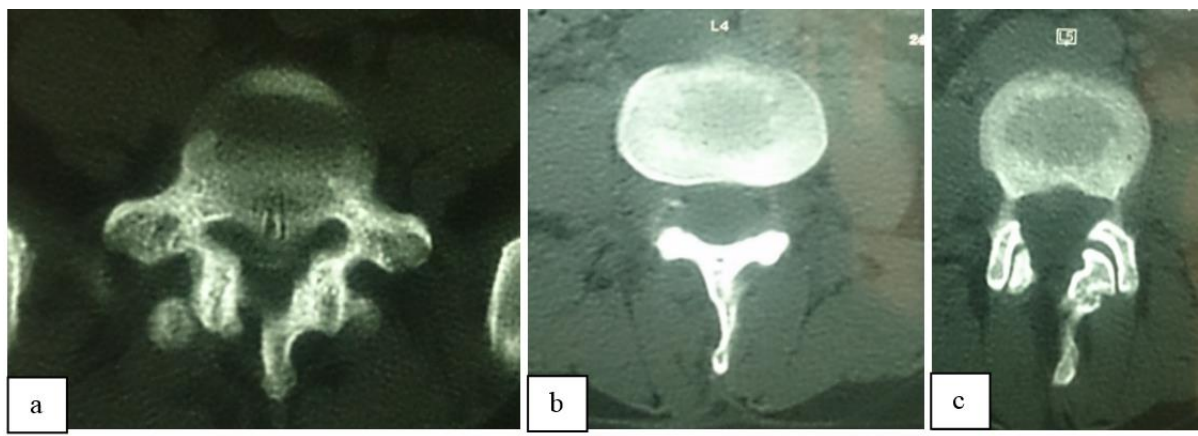

Fig. 8

A standard laminectomy was performed, ligament flavum was excised, and any offending osseous or discogenic compressing material was removed to achieve adequate decompression of the entire stenotic width and length of the dural sac and its contents. Deroofing of the nerve root canals was performed when required. Osteotomized spinous process along with their attachment repositioned, dead space avoided by approximating paraspinal muscle by intermittent suturing. Dorsolumbar fascia closed in continous manner. The wound was closed in layers over the suction drain. No blood transfusion was used for decompression up to 3 levels. The drain was removed after 48 hours and the patients were encouraged to walk with a light brace on 3rd or 4th postoperative day. The spinal brace was gradually discarded about 3 months after the operation. All physical and routine activities allowed to all patients after 3 months of the operation. followup were included in the study.

\section{Results}

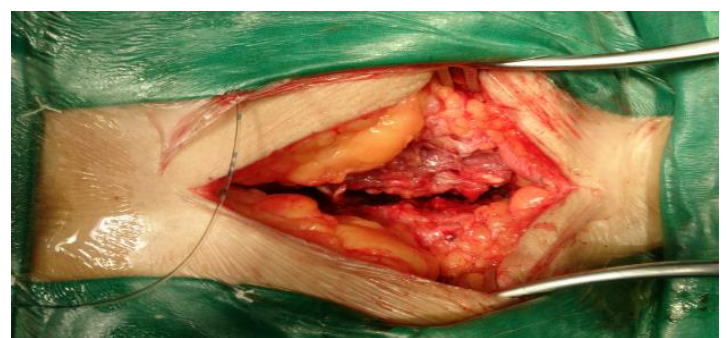

Fig. 7

On follow-up, the patient and his/her close relations were enquired about any changes in the posture while walking, increase in the walking distance, improvement in dysesthesia in lower limb, the motor power, capability to climb stairs. The patients were called 2 weeks after discharge from the hospital for clinical assessment and stitch removal. They were evaluated at 6 weeks, 3 months after the procedure, and thereafter at 1 year. Patients were followed up by periodic clinical examination, assessment of VAS and ODI score. X-rays anteroposterior and lateral view of lumbosacral spine in maximum flexion and extension was taken on each follwup to assess the instability and CT scan at 1 year to assess the union of osteotomized spinous process. All patients with minimum 18 months

Total 20 patients were included in our study which could be followed up for 18 months or more were 
included in this study. The average age at surgery for male was 54.3 years and for female 51.7 years. The average follow-up was 11.5 months. Eleven (55\%) patients had decompression at single level, five patient (25\%) had 2 level and four patients had more than 2 level (40\%) decompression. Bilateral decompression done in $75 \%$ patient and unilateral decompression done in $25 \%$ patients. L4 MSPO done for all patients, L2 in $5 \%(1 / 20)$, L3 in $40 \%(8 / 20)$ and L5 in $80 \%(16 / 20)$ patients.

Intraoperative complications were happened in only 1 patient in the form of dural tear which was repaired without any further odd consequences. No post operative complication occurred in any of our patients. $95 \%$ pts ambulated on day one. $75 \%$ resumed normal work at 3 weeks. There was no clinical or radiological evidence of instability at 1 year followup. $70 \%(14 / 20)$ showed spinous process union with retained laminar bridge at 1 year.

Result of this study is shown in Table 2. The overall result of our study were good to excellent in $85 \%$ (17 patients), fair result in 15\% (3 patients) and no poor result at 6 months follow-up. At 1 year follow-up 93\% patient had good to excellent result. The average preoperative and postoperative VAS score for back and leg pain is shown in Table 3.90\% improvement in VAS for back pain and $100 \%$ improvement in VAS for leg pain seen after MSPO technique in our study. ODI also improved significantly after MSPO technique (Prop average ODI 72, Post op average ODI 22.6).

Table 2

\begin{tabular}{|l|c|c|}
\hline & 6 Month Follow-up & 1 Year Follow-up \\
\hline Good to Excellent & $85(17 / 20)$ & $93(18 / 20)$ \\
\hline Fair & $15(3 / 20)$ & $7(2 / 20)$ \\
\hline Poor & 0 & 0 \\
\hline
\end{tabular}

Fig. 9. Full flexion at 1 year

At final follow up at 1 year all patients were satisfied with the procedure with no difficulty in doing active flexion-extension movement (Fig. 9 and 10), active lateral bending and rotations smoothly, without "catch" or apprehension. Patients experienced marked relief (70-90\%) from neurogenic claudication and they observed marked improvement in their preoperative stooping posture.

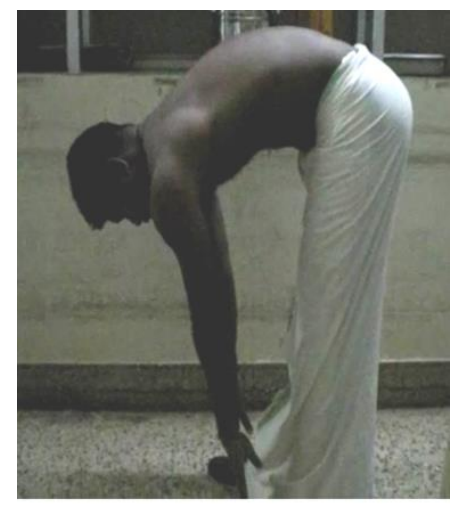

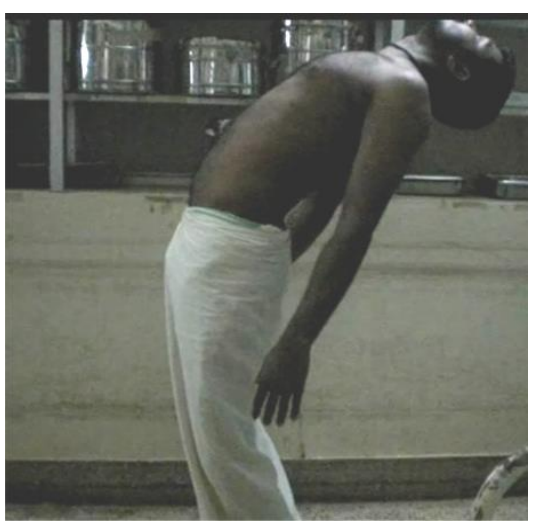

Fig. 10. Full extension at 1 year

Table 3

\begin{tabular}{|l|c|c|c|}
\hline & Pre op Average (M,F) & Post op Average (M,F) & Improvement \\
\hline VAS-Back Pain & $7.4(7.4,7.6)$ & $3.3(3.3,3)$ & $90 \%$ \\
\hline VAS -Leg Pain & $7.75(7.8,7.5)$ & $1.8(1.8,1.5)$ & $100 \%$ \\
\hline ODI & $72(72,70)$ & $22.6(22.6,21)$ & \\
\hline
\end{tabular}

Comparison of VAS at 3 months and ODI at 1yr gave similar results.

\section{Discussion}

Conventional laminectomy technique exposures of lumbar posterior elements, which include stripping of multifidus bilaterally, with subsequent wide resection, have potentially serious consequences. In Massshi et $\mathrm{al}^{11}$ study they mentioned that commonly used techniques of lumbar decompression that Include bilateral elevation of paraspinal musculature and Aggressive bony resection can result in significant iatrogenic instability of spine. Mayer et $\mathrm{al}^{12}$ demonstrated a decrease in muscle strength with associated atrophy on postoperative computed 
tomography. In Bradley $\mathrm{K}$ Weiner et $\mathrm{al}^{13}$ study, they found that use of spinous process osteotomies in lubar decompressive surgery provides excellent visualization and room to work while minimizing resection and injury to tissues not directly involved in the pathological process. To overcome the iatrogenic instability created by conventional laminectomy and need for posterior stabilization and fusion of mobile segments of the spine entails hypermobility of the adjacent unfused joints which may lead to instability or premature disc degeneration or stenosis of the canal a less invasive newer technique is required. The incidence of adjacent segment degeneration in patients followed up for 5 years or more after fusion has been reported as $10-30 \% .{ }^{14-16}$ Such changes may reflect the natural history of lumbar spondylosis over the years. ${ }^{17,18}$ The recent changes in minimally or less invasive spine surgery techniques has been based on the goals of minimization of destruction to unaffected tissues and optimisation of the cosmetic results with adequate exposure to decompress the thecal sac and nerve root. These goals should be to achieve decompressive surgery completely and safely. Microendoscopic techniques involve unilateral paraspinal retraction, ipsilateral decompression, and contralateral decompression, using microendoscope and under the midline posterior structures. However, the ipsilateral portion of this technique is limited by visualisation and Kerrison angulation of the lateral zone leads to inadequate decompression on canal and nerve root.

In our new technique of modified spinous process osteotomy appears to achieve these goals, while affording excellent visualisation and complete decompression. MSPO preserves the posterior midline elements while allowing them to be retracted away from the working area, and it also respects the integrity of the spinous processes and supra-/interspinous ligaments while removing the ligamentum fravum and decompressing the thecal sac and lateral recess and our study was comparable with the Masashi et al ${ }^{11}$ study. In our study intra-op complication occur in the form of dural tear with kerrison rongeur which was repaired without any odd incidence. In Masashi et al study they had 2 dural tear.

The process of MSPO can be accomplished with less surgical exposure and complexity, translating into decreased blood loss and operating time, with no major complication. Furthermore, the use of MSPO does not require any specific instruments or extensive experience.

\section{Conclusion}

Modified spinous process osteotomy is less invasive, less techniqually demanding and a simple procedure without any serious complications. Multilevel lumbar canal stenosis of any variety (developmental, degenerative or combined variety), with or without concomitant intraspinal pathology can be decompressed satisfactorily by this novel technique. The retained median structures maintain the tension band and the strength and possibly the proprioceptive sensations of the lumbar spine, which ensure least disturbance of kinematics, mobility, stability and lordosis of the operated lumbar spine and concomitant discectomy, root-canal deroofing can be done comfortably when indicated.

\section{Source of Support: Nil.}

\section{Conflict of Interest: None.}

\section{References}

1. Bosworth DM, Filding JW, Demarest L. Bonequist, A case of spondylolisthesis treated by arthrodesis. J Bone Jt Surg. 1955;37A:767-786.

2. Arnoldi CC, Brodsky AE, Cauchoix L, Crock HV, Dommisse GF, Edgar MA, et al. Lumbar spinal stenosis and nerve root entrapment syndrome: Definition and classification. Clin Orthop Relat Res. 1976;115:4-5.

3. Weinstein J.N, Tosteson T.D, Lurie J.D, Tosteson A, Blood E, Herkowitz H, et al. Surgical versus nonoperative treatment for lumbar spinal stenosis fouryear result of the Spine Patient Outcomes Research Trial. Spine (Phila Pa 1976) 2007;35,1329-1338.

4. Leone A, Guglielmi, G, Cassar- Pullicino, V.N. Bonomo L. 2007. Lumbar intervertebral instability: a review. Radiology. 2007;245:62-77.

5. Verbiest H. Results of surgical treatment of idiopathic developmental stenosis of the lumbar vertebral canal; A review of twenty-seven years experience. $J$ Bone joint Surg Br. 1977;59;181-8.

6. Lee CK. Lumbar spine instability (olisthesis) after extensive posterior spinal decompression. Spine (Phila Pa 1976). 1983;8:429-3.

7. Frymoyer JW, Henley EN Jr, Howe J, Kuhlmann D, Matteri RE. A comparision of radiolographic findings in fusion and nonfusion patient ten or more years following lumbar disc surgery. Spine (Phila Pa 1976)1979;4:43540.

8. Lehmann TR, Spratt KF, Tozzi JE, Weintein JN, Reinarz SJ, el-Khoury GY, et al. Long-term follow-up of lower lumbar fusion patients. Spine (Phila $\mathrm{Pa}$ 1976).1987;12:97-104

9. Lee CK. Accelerated degeneration of the segement adjacent to lumbar fusion. Spine (Phila $\mathrm{Pa}$ 1976).1988;13:375-7

10. Arnoldi CC, Brodsky AE, Cauchoix J, Crock HV, Dommisse GF, Edgar MA, et al. Lumbar spinal stenosis and nerve root entrapment syndromes: Definition and classification. Clin Orthop Relat Res. 1976;115:4-5

11. Masashi Takaso \& Toshiyuki Nakazawa \& Takayuki Imura, Less invasive and less technically demanding decompressive procedure for lumbar spinal stenosisappropriate for general orthopaedic surgeons? International Orthopaedics (SICOT). 2011;35:67-73.

12. Mayer TG, Vanharanta H, Gatchel RJ. Compparision of CT scan muscle measurements and isokinetic trunk strength in postoperative patients; Spine. 1989;14:33-6.

13. Bradley K. Weiner, Robert D Fraser. Spinous process osteotomy to Facilitate Lumbar Decompressive Surgery. Spine: January $1^{\text {st }}, 1999 ; 24(1): 62-66$.

14. Frymoyer JW, Henley EN Jr, Howe J, Kuhlmann D, Matteri RE. A comparison of radiographic findings in 
fusion and nonfusion patients ten or more years following lumbar disc surgery. Spine (Phila Pa 1976).1979;4:43540.

15. Lehmann TR, Spratt KF, Tozzi JE, Weintein JN, Reinarz SJ, el-Khoury GY et al. Long- term follow-up of lower lumbar fusion patients. Spine (Phila Pa 1976).1987;12:97-104.

16. Lee CK. Accelerated degeneration of the segement adjacent to lumbar fusion. Spine(Phila Pa 1976). 1988;13:375-7.

17. Johnson KE, Rosen I, Uden A. The natural course of lumbar spinal stenosis. Clin Orthop. 1992;279:82-6.

18. Spivak J. Degenerative lumbar spinal stenosis. J bone Joint Surg Am. 1998;80:1053-66.

How to cite this article: Chandan K, Vellara JF, Bamb HB, Jai KKS, Sharma A. Functional and radiological outcome of modified spinous process osteotomy (MSPO) technique in decompressive surgery for lumbar canal stenosis. Indian J Orthop Surg. 2018;4(3):234239. 